\title{
The Barkhausen Method to Investigate Powerful Processes during Action of Piezoelectric Igniters
}

\author{
Linas ARDARAVIČIUS, Skirmantas KERŠULIS, Oleg KIPRIJANOVIČ *, \\ Česlovas ŠIMKEVIČIUS, Steponas AŠMONTAS
}

\author{
Center for Physical Sciences and Technology, Vilnius, Sauletekio al. 3, LT-10257, Lithuania \\ crossref http://dx.doi.org/10.5755/j01.ms.26.3.23320
}

Received 03 May 2019; accepted 20 November 2019

\begin{abstract}
The Barkhausen method is proposed to clarify the cause of radiation of electromagnetic (EM) pulses during high voltage pulse generation by piezoelectric igniters (PIs). Wide bandwidth of the experimental setup was narrowed for a simultaneous registration of electric and detected EM pulses by a two-channel oscilloscope. The PI was loaded on a high ohmic resistance and high voltage pulses of $8-17 \mathrm{kV}$ amplitude and up to $150 \mu \mathrm{s}$ in total duration were registered. These pulses contained a series of short pulses called Barkhausen type pulses. Duration of these pulses having the relatively high amplitude was 30-40 ns. The registration revealed that the radiating EM pulse series corresponded to Barkhausen type pulse series. Short non-radiating negative pulses appearing during the saturated voltage growth were also observed and they had relaxation tails. The analysis showed that the EM pulses are caused as a result of domain switching with high voltage spikes at the PZT cylinders bases, where high electric fields are created. The activity of these switchings weakens when the "age" of PIs increases. The non-radiated pulses resulted from fast internal screening processes in the volume of the cylinders, accompanied by the impact ionization. The increase of the saturation and PI's "age" causes lengthening of the relaxation tails. The results of practical importance for PIs in monitoring systems are placed. It is concluded that the Barkhausen method in wideband configuration is a convenient experimental arrangement for investigation of powerful processes in ferro-piezoelectric ceramics.

Keywords: piezoelectric ignition, Barkhausen method, Barkhausen type pulses, series of EM pulses, surface domain switching.
\end{abstract}

\section{INTRODUCTION}

A paper entitled "Two phenomena discovered with the help of new amplifiers" was published by H. Barkhausen 100 years ago. There were investigated ferromagnetic specimens by placing them in a repolarized magnetic field and registering an induced electric signal. The signal was detected with the aid of coil and then it was amplified [1]. A noise-like signal consists of series of voltage pulses induced in the coil placed close to the surface of the ferromagnetic specimen. Crackling sounds registered from the specimen were later called Barkhausen noises and Barkhausen clicks or jerks. Such electric signal reflected jump-like dynamics of an internal domain structure - called the Barkhausen effect. Soon after that, the mechanical Barkhausen effect was revealed. This was a possibility to observe the appearance of jump-like behavior of domains which was induced by compressing the specimens [2]. For a relatively long time, it was believed that this effect can mainly serve only as a demonstration of the domain structure existence in ferromagnetics.

Ferroelectric materials also contain domains and later, in the $40 \mathrm{~s}$, the Barkhausen method was applied to them. The oscilloscopes started to be used for registration of signals from the samples under repolarized electric fields. During repolarization in ferroelectrics, rescreening can be provided by movement of charges both through the internal and the

\footnotetext{
* Corresponding author. Tel.: +370-5-2648884; fax: +370-5-2649774

E-mail address: oleg.kiprijanovic@ftmc.lt (O. Kiprijanovič)
}

external circuits. Therefore, in experiments specimens in capacitor form also were used [3].

Unlike to magnetism, in ferroelectrics free charges screen electric fields created by spontaneous polarization. Hence, the dynamics of domain switching has its own specifics [4-6]. Modification of the method when domain dynamics is induced by temperature changes was introduced in the beginning of 50s. This modification of method was applied in the investigations as thermal Barkhausen effect method [7]. The Barkhausen method became a famous tool because it is distinguished by its high sensitivity to observe fast processes which appear when the repolarization processes of ferromagnetic and ferroelectrics takes place $[8,9]$.

Lead zirconate titanate (PZT) ferroelectric ceramics with piezoelectric properties is used in piezoelectric igniters (PI). Complexity of the ceramics having grain and domain structures with boundaries of different properties complicate their investigation, especially, when devices are in action [10-12]. Recent progress in measuring electronics made investigation of complex long-continued signals more convenient [13]. Therefore, broadband digital oscilloscopes and computer software allow to obtain the today necessary results using long known investigation methods or their modifications.

We registered electromagnetic (EM) pulses using a wideband horn antenna and a wideband oscilloscope when PIs were activated [14]. Multiple series of short EM pulses 
were found, which differ by duration and by pulse amplitudes and some difficulties to register them occur. The duration of a single EM pulse is 3-5 ns.

It was only clear that radiation of EM pulses was the result of fairly powerful processes when high voltage values by PIs are reached in a time of few $\mu s[15,16]$. The main problem of simultaneous measurements of the EM pulses with high voltage pulse is a high value of the pulse amplitude. It was investigated the action of the commercially available PIs on high ohmic load in order to disclose an appearance of EM pulse series. Our experimental arrangement is very similar to Barkhausen circuit, but instead the coil we use logoperiodic antenna with detection of EM pulses, which and the divided electric signal were registered by two channel oscilloscope.

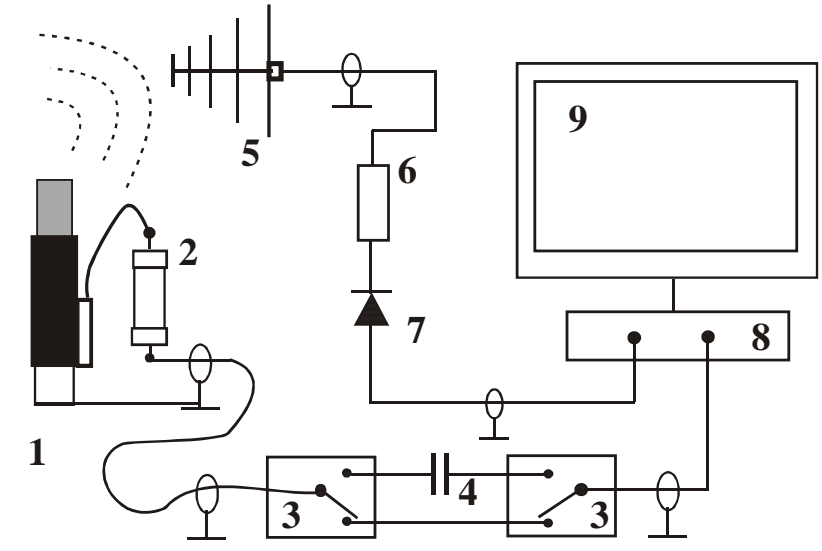

Fig. 1. Schematic diagram of the experimental setup: 1-PI; 2 -upper shoulder of resistive divider; 3-broadband switches; 4 -transfer capacitor; 5 -logoperiodic television range antenna; 6-75-50 $\Omega$ transition; 7-broadband detector; 8 -two channel digitized oscilloscope; 9-PC

\section{EXPERIMENTAL}

The piezoelectric rigid cylinders of PIs are sintered from PZT ferroelectric ceramics at $1100-1300{ }^{\circ} \mathrm{C}$ temperature. PZT cylinders just after sintering have statistically distributed orientation of domains. The cylinders are mainly composed of $\mathrm{PbZr}_{0.52} \mathrm{Ti}_{0.48} \mathrm{O}_{3}$ with tetragonal symmetry and the $\mathrm{Zr}: \mathrm{Ti}$ proportion of the composition are close to morphotropic transition $\mathrm{Zr}: \mathrm{Ti}=55: 45$ [10]. During the poling, the cylinders are exposed to a strong electric field that causes the electric dipoles to become aligned in the direction of the field, creating remnant polarization. Such ceramics for strike igniters near the transition has dynamic electric permitivity $\varepsilon=2000$, quality $Q$ factor is only $60-75$, loss factor $\tan \delta$ is $15-20 \times 10^{-3}$. Piezoelectric activity coefficient $d_{\mathbf{3 3}}$ is about $400 \times 10^{-12} \mathrm{C} / \mathrm{N}$ and coupling factor $k_{p}$ lies in the range within $0.59-0.62$. Number of ignitions by PI is about $1.2 \times 10^{4}$ times. Construction of the PI where two cylinders are in parallel with opposite remnant polarization is described in Ref. [14].

In order to simplify signal registration where the shortest are EM pulses of 3-5 ns and the longest is high voltage pulse of tens $\mu$ s in durations, a logoperiodic antenna with detector and an oscilloscope with a lower bandwidth than in Ref. [14] were used.
A schematic of experimental setup is presented in Fig. 1. It consists of PI (1), an upper resistive divider shoulder (2), a broadband switches (3), a transfer capacitor (4), a television range antenna (5), a 75-50 Ohm transition (6), a broadband detector (7), an digitized oscilloscope of 0 $150 \mathrm{MHz}$ bandwidth (8) and a personal computer (9). To visualize the high voltage pulse after generation the divider (2) with a high division coefficient is used. The upper divider shoulder (2) is a load of the PI which has a value of $0.5 \mathrm{M} \Omega$ or $1 \mathrm{M} \Omega$ and the lower shoulder is the $50 \mathrm{Ohm}$ oscilloscope input. The upper shoulder is made from one or several thin film cannular resistors of total $1.5-2.5 \mathrm{~cm}$ in length. It was established that from $400 \mathrm{k} \Omega$ and up to $1 \mathrm{M} \Omega$ there exists repeatable generation of high voltage pulses by "fresh" and "aged" PIs. The lower shoulder is $50 \Omega$ oscilloscope input and, therefore, the voltage is divided by $1: 1 \times 10^{4}$ to $1: 2 \times 10^{4}$. However, it is practically impossible to construct the resistive divider with smooth amplitudefrequency response for such resistance values. The construction depends mainly on used resistor models and construction of the divider. If the high voltage pulse of $\mu \mathrm{s}$ duration has the coefficient the same as mentioned values, then for short, Barkhausen type pulses, their amplitude values are higher by $10-15 \%$. The divided pulse transmitted to the second oscilloscope channel by $50 \Omega$ wave resistance cable. The switches (3) set the second channel through the transfer capacitor (4) of $10-12 \mathrm{pF}$ and serves as a filter of low frequencies. Radiated signal is transmitted to the first oscilloscope channel. The obtained oscillograms can be processed by computer software.

\section{RESULTS}

Piezoelectric forces generate a positive charge on bases of the cylinders near the high voltage electrode, which then relaxes with sign inversion (see Fig. 2).

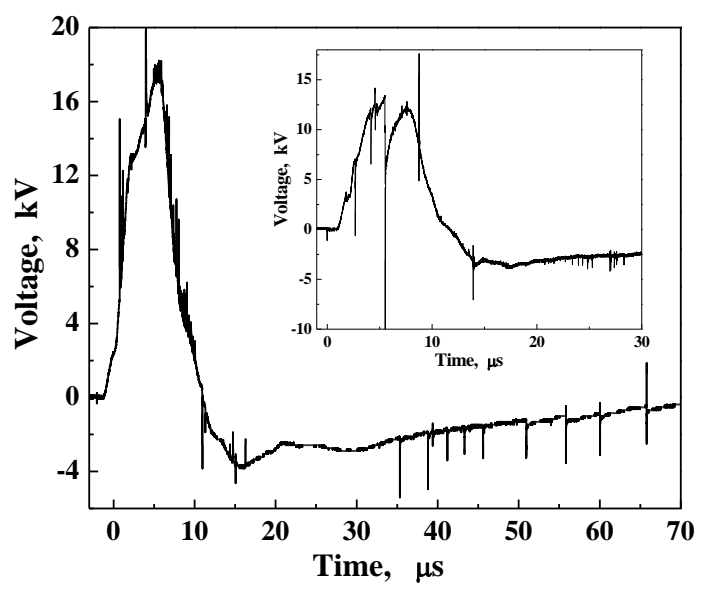

Fig. 2. View of the high voltage pulse with short Barkhausen type pulses generated by a "fresh" PI loaded at $1 \mathrm{M} \Omega$ resistance. Inset represents an oscillogram of the high voltage pulse for "aged" PI loaded at $1 \mathrm{M} \Omega$ resistance

Voltage growth has linear section of $2.5-3 \mu \mathrm{s}$ in duration and non linear saturation lasts a few $\mu$ s up to maximal value and almost linear voltage droop. The main 
part of the high voltage pulse lasts about $10 \mu$ s and then it relaxes and the visible relaxation takes part up to $70 \mu \mathrm{s}$.

The proposed method let us to observe columns of different polarity. They represent short electrical pulses of $30-40 \mathrm{~ns}$ in duration, and so these pulses are seen at this scale as columns.

For "aged" PI (see inset in Fig. 2), the generated high voltage pulse has a lower amplitude value. Also, the decrease in number the short pulses and the appearance of negative pulses with relaxation tails are observed. The pulse with relaxation tail starting at $6 \mu$ s can even be called colossal or giant.

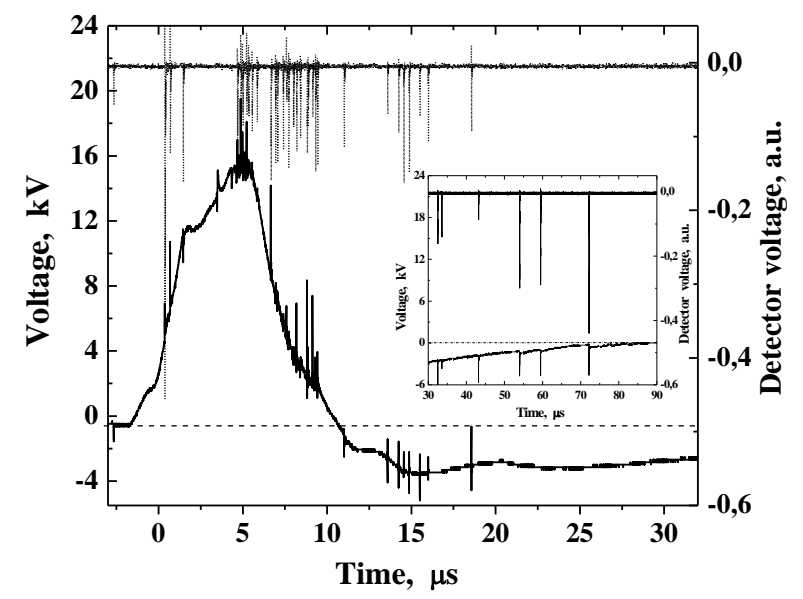

Fig. 3. Simultaneous registration of the high voltage pulse and the detected EM pulses. Inset shows relaxation of the pulse with simultaneously detected EM pulses

The structure of the high voltage pulse roughly can be divided into sections: 1 -starting linear voltage growth $0-2.5-3 \mu \mathrm{s} ; 2-3-6.5 \mu \mathrm{s}$ non-linear saturation of the high voltage; 3 -short maximum of the pulse; 4 -approximately linear high voltage droop 7-12 $\mu$ s; 5-sign inversion and transition to relaxation $12-30 \mu$ s and 6 -relaxation of the pulse $30-100 \mu$ s or for some PIs even up to $150-\mu$ s. Inset in Fig. 3 shows the relaxation part of the pulse where electromagnetic pulses are also simultaneously detected.

We assign the term Barkhausen type pulses to the observed short electrical pulses. As one can see in Fig. 3, almost all Barkhausen type pulses which are present through the high voltage pulse radiate EM pulses.

Therefore, detected EM pulses, which correspond to them, are also Barkhausen type pulses. EM pulses are emitted in series and we enumerate these series according to the appearance of the series of Barkhausen type pulses. First series appears just after the pulse beginning during linear voltage growth and has positive pulses only; the second series appears at the top of the pulse where the pulses of both polarities often are present; the third is observed during the pulse droop, where mostly positive pulses are, the fourth, comparatively short, but intense with pulses of both polarities; and the fifth, the longest, but with rarely repeated, mostly negative, pulses. One can notice single positive pulse at $4 \mu \mathrm{s}$ having relaxation tail, but without accordingly detected EM signal.

We cannot present variety of sweep oscillograms and discuss differences between series of the Barkhausen type pulses in this paper. Here we decided to present two figures that are relating to the practical applications of PIs. Fig. 4 presents the beginning of the three oscillograms where pulses were generated by PIs with different numbers of ignitions.

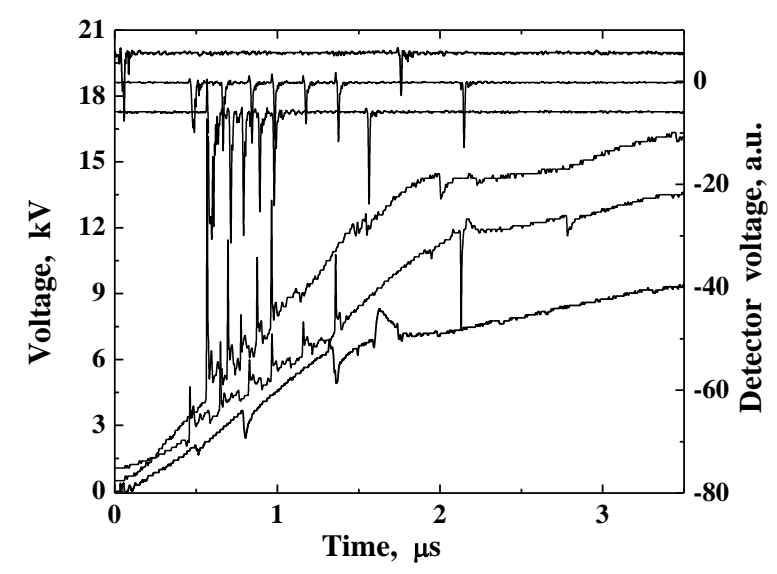

Fig. 4. Beginning (up to $3.5 \mu$ s) of high voltage pulses generated by PIs which differ on their "age" characteristics. Simultaneously measured signals of the detector are placed on the top. The highest voltage generates the "fresh" PI. For clarity, signals of the detector are in the reverse order

The curve having the highest voltage is generated by "fresh" PI, the middle one by PI that had about half of $1.2 \times 10^{4}$ guaranteed ignitions and the lowest curve by "aged" PI which had more than the half of the guaranteed ignitions. The detector signals are also present on the top. One can see the decrease of high voltage amplitude with an increase in the number of ignitions. Also, the decrease of amplitudes of the first series is observed. On another hand, non-radiating negative pulses for the lowest curve have increased amplitudes with increased relaxation tails.

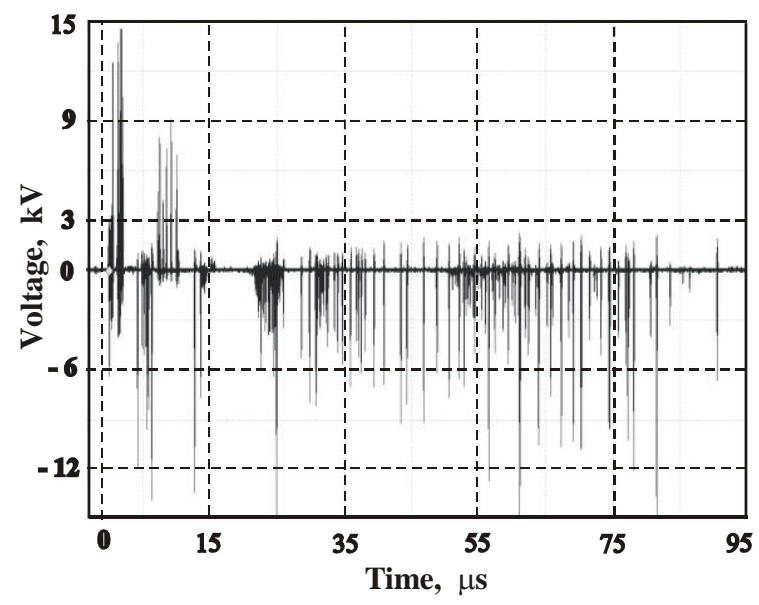

Fig. 5. Barkhausen view oscillogram (through the transfer capacitor) of the PI with high intensity of Barkhausen type pulses

Our attention was drawn to PI that generated the high voltage pulse with increased intensity of Barkhausen type pulses having large enough amplitudes. But generated high voltage pulse had amplitude of $8 \mathrm{kV}$ only. Fig. 5 
demonstrates this oscillogram obtained using the transfer capacitor.

\section{DISCUSSION}

When we use the divider and the antenna with detector without an amplifier, we can hardly expect to observe Barkhausen pulses and noises [17, 18]. Instead, we assign the term Barkhausen type pulses to short pulses after the divider, which appear through the spanning of the registered high voltage pulse, and to detected EM pulses which correspond to short electrical pulses.

Simultaneous registration of the high voltage pulse and detected EM pulses reveal that emitting Barkhausen type pulses appear through almost all high voltage pulse sections. The only exception is nonlinear voltage growth section and there exactly appear pulses not radiating EM pulses (see Fig. 3, Fig. 4). It becomes immediately clear that these spikes are responsible for the emission of EM pulses. These relatively high voltage spike series emitting EM radiation, according to the description, are the results of powerful surface processes where electric field strength can reach $3 \times 10^{6} \mathrm{~V} / \mathrm{cm}$. Therefore, these spikes can induce electron emission from the surface [19]. Moreover, they are the consequence of domain switching influenced by electronic properties of crystallite surfaces and are generated on the cylinder contact surface area - as a result, they are superimposed upon the high voltage pulse [4,20]. The emission is intensively investigated (although its origin is not completely uncovered) because it is important in practical applications [21]. However, to apply the emission currents in practice a cathode of special construction must be made.

An unscreened non-compensated poly-domain structure must be formed in the ceramics for activation of the powerful processes. The ways of the formation can be: a) by fast polarization reversal to apply an external field, b) by piezoelectric effect, as we have, and c) by other methods including a paraelectric-ferroelectric phase transition $[22,23]$. During the unscreening process a spontaneous polarization temporally appears and the potential of occurring surface charge deforms the surface barrier in such way that both tunneling of electrons and generation of the spikes become possible. This is partly confirmed by spikes disappearance with the increase of number of ignitions and with the cylinder contact wearing (see Fig. 4).

The reverse, i.e. positive pulse at $4 \mu$ s in Fig. 3 has no adequate detected EM pulse. The Barkhausen pulses of reverse polarity often are observed through the experiments [19]. The reversely appeared pulses are one more demonstration of the switched domain structure, where differently directed domains appear. As it follows from Fig. 4, such pulses without EM pulses should be negative and with relaxation tails that are the longer the higher the voltage is. So, they correspond to another powerful process. It was ascertained that during repolarization by short pulses fast rising internal electrical fields of space charges are formed in $\mu$ s time scale and, therefore, they have no diffusive origin [16]. Such powerful process can be linked to the fast internal screening which is disclosed by the presence of relaxation tails [24]. A high local electric field strength between the domain boundaries induces the impact ionization generating free carriers from deep levels accompanied by the optical radiation during the relaxation $[6,24]$. These impacts restrict the linear high voltage growth. We can't decide whether we define the pulses with the relaxation tail as Barkhauzen type pulses because they are initiated by a process of another nature.

It remains unclear how the voltage growth influences the surface area causing cessation of the spikes generation after the linear growth. The piezoelectric forces are active up to the top of the pulse and the reappearance of the spikes in the second series at the top remains unclear, although it is strongly associated with the reduction of the piezoeffect. The first series has structure to which quasi period can be ascribed. The second series at the top of the high voltage pulse are more similar to Barkhausen pulse series, which have rather random appearance [25]. But in contrast to Barkhausen pulse series, the second and fourth ones have a large quantity of the reverse pulses.

PIs, as well as piezoelectric mechanisms that react to stress, due to their autonomy and sufficient durability are used as sensors in monitoring systems. It is possible to transmit acoustic, optical, electrical, and EM signals generated by PIs in various combinations through different channels. For example, PI emits EM pulses of increased density (Fig.5), which can be received by the antenna, while generated electrical Barkhausen type pulses could be transmitted by a cable.

\section{CONCLUSIONS}

The old method proposed 100 years ago was used in a wideband modification to determine the appearance of EM pulse series accompanied by the PIs action. The PI was loaded on a high ohmic resistor and the use of television antenna with a detector aimed to narrow the bandwidth allowed a simultaneous registration of the electric and detected EM pulses by a two-channel oscilloscope.

There were observed 30-40 ns duration Barkhausen type pulses which appeared in series during a $100 \mu \mathrm{s} \mathrm{high}$ voltage pulse. These series emitting EM pulses are present in all sections of the high voltage pulse, with the exception of saturated voltage growth, and are associated with powerful processes near the high voltage electrode. These processes are as a consequence of surface domain switching at high electric fields. During nonlinear high voltage saturation the revealed pulses are non-radiating EM pulses having relaxation tails. They are the result of powerful processes restricting the high voltage growth including the impact ionization with generation of free carriers.

Some results show the features when PIs can be used as sensors in monitoring systems. So, the modification of Barkhausen method can be used to investigate devices containing elements from ferroelectrics with piezoeffect. Widening of the arrangement bandwidth gives more detailed information about short powerful processes during the high voltage generation and the relaxation processes.

\section{REFERENCES}

1. Barkhausen, H. Zwei mit Hilfe der neuen Verstärker entdeckte Erscheinungen Physische Zeitschrift 20 1919: pp. $401-403$. 
2. Gerlach, W., Lertes, P. Über Magneto-elastische Effekte Zeitschrift für Physik 4 1921: pp. 383-392.

3. Lee J.K., Shin, G.Y., Song, K., Choi, W.S., Shin Y.A., Park, S.Y., Britson, J., Cao, Y., Chen, L.Q., Lee, H.N., Oh, S.H. Direct Observation of Asymmetric Domain Wall Motion in a Ferroelectric Capacitor Acta Materialia 61 2013: pp. $6765-6777$. http://dx.doi.org/10.1016/j.actamat.2013.07.051

4. Chynoweth, A. G. Surface Space-Charge Layers in Bariuiin Titanate Physical Review 102 1956: pp. 705-715.

5. Genenko, Y. A., Hirsch, O., Erhart, P. Surface Potential at a Ferroelectric Grain due to Asymmetric Screening of Depolarization fields Journal of Applied Physics 115 2014: pp. 104102-1-104102-13.

http://dx.doi.org/10.1063/1.4867984

6. Sadykov, S., Turik, A. Electroluminescence and Internal Screening of Domains upon Polarization Switching of Ferroelectric Ceramics in a Pulsed Self-Consistent Field Physics of the Solid State 45 (11) 2003 pp. 2178-2182. https://doi.org/10.1134/1.1626759

7. Bol'shakova, N., Zaznobin, T., Ivanov, V., Murav'eva, E., Ped'ko, B. The Barkhausen Thermal Effect as Applied to Investigation of the Polarization Switching of Barium Strontium Niobate Crystals Physics of the Solid State 48 2006: pp. $1028-1029$. https://doi.org/10.1134/S1063783406060035

8. Lopez, R. J. Simultaneous Observation of Barkhausen Pulses and Jumps in Resistance Fluctuations in Ferromagnetic Materials Review of Scientific Instruments 70 (1) 1999: pp. $171-174$. https://doi.org/10.1063/1.1149560

9. Chynoweth, A. G. Effect of Space Charge Fields on Polarization Reversal and the Generation of Barkhausen Pulses in Barium Titanate Journal of Applied Physics 30 (5) 1959: pp. 280-285.

10. Okazaki, K. Engineering for Dielectrics. 4th ed. Gakkensha, Tokyo, 1992.

11. Cross, L. E. Ferroelectric Ceramics: Tailoring Properties for Specific Applications; in: Ferroelectric Ceramics. eds Setter, N., Colla, E. L. Birkhäuser, Basel, Boston, Berlin, 1993: pp. $1-86$.

12. Kamlah, M. Ferroelectric and Ferroelastic PiezoceramicsModeling of Electromechanical Hysteresis Phenomena Continuum Mechanics and Thermodinamics 13 (4) 2001: pp. 219-268.

13. Reid, A., Judd, M. Ultra-wide Bandwidth Measurements of Partial Discharge Current Pulses in $\mathrm{SF}_{6}$ Journal of Physics D: Applied Physics 40 2007: pp. 2948-2953. https://doi.org/10.1088/0022- 3727/45/16/165203

14. Anisimovas, F., Ašmontas, S., Kiprijanovič., O., Maneikis, A., Vengalis, B. Broadband Electromagnetic Emission from PZT Ferroelectric Ceramics after Shock Loading Materials Science (Medžiagotyra) 19 (4) 2013: pp. $433-437$. http://dx.doi.org/10.5755/j01.ms.19.4.3137

15. Rosenman, G., Shur, D., Krasik, Ya. E., Dunaevsky, A. Electron Emission from Ferroelectrics Journal of Applied. Physics 88 (11) 2000: pp. 6109-6159. https://doi.org/ 10.1063/1.1319378

16. Sadykov, S. Borodin, V. Agalarov, A. Reversible Polarization of Ferroelectric Ceramics in a Rapid-growing Electric Field Technical Physics 45 (6) 2000: pp. $776-779$.

17. Tan, C. D., Gargner, J., Morrison, F. D., Salje, E. K. H., Scott, E. F. Studies of Barkhausen Pulses in Ferroelectrics arXiv:1805.08536 [cond-mat.mtrl-sci] 2018: pp. 1-48.

18. Salje, E. K. H., Dahmen, K. A. Crackling Noise in Disordered Materials Annual Review of Condensed Matter Physics 5 2014: pp. 233-254.

https://doi.org133838/ 10.1146/annurev-conmatphys031113-133838

19. Miller, R. On the Origin of Barkhausen Pulses in $\mathrm{BaTiO}_{3}$ Journal of Physics and Chemistry of Solids $17(1,2)$ 1960: pp. $93-100$.

20. Pavlov, A., Raevskii, I., Sakhnenko, V. Electron Emission during Pulsed Polarization Switching of Ferroelectric Ceramics Technical Physics 44 (7) 1999: pp. 782-785.

21. Krasik, Ya. E., Chirko, K., Sayapin, A., Gleizer, J., Krokhmal, A., Felsteiner, J. Electron Beam Generation in a Diode with Different Ferroelectric Cathodes Applied Physics Letters 94 (8) 2003: pp. 5158-5162. https://doi.org/10.1063/1.1611628

22. Benedek, G., Boscolo, I., Handerek, J., Marchesini, S., De Martinis, C., Riege, H., Scurati, A. Displacement and Emission Currents from PLZT 8/65/35 and 4/95/5 Excited by a Negative Voltage Pulse at the Rear Electrode Nuclear Instruments and Methods in Physics Research Section A: Accelerators, Spectrometers, Detectors and Associated Equipment 393 1997: pp. 469-473. https://doi.org/10.1016/S0168-9002(97)00548-2

23. Likodimos, V., Orlik, X. K., Pardi, L., Labardi, M., Alegrini, M. Dynamical Studies of the Ferroelectric Domain Structure in Triglycine Sulfate by Voltage-modulated Scanning Force Microscopy Journal of Applied Physics 87 2000: pp. $433-451$. https://doi.org/10.1063/1.371882

24. Sadykov, S. A., Agalarov, A. Sh., Kallaev, S. N. Switching Induced Electroluminescence in PZT Ceramics in HighVoltage Pulse Field Integrated Ferroelectrics 72 2005: pp. $27-30$. https://doi.org/10.1080/10584580500312586

25. Zhukov, S., Genenko, Y. A., Hirsch, O., Glaum, J., Granzow, T., von Seggern, H. Dynamics of Polarization Reversal in Virgin and Fatigued Ferroelectric Ceramics by Inhomogeneous Field Mechanism Physical Review B 82 2010: pp. 014109-1-014109-8 https://doi.org/10.1103/PhysRevB.82.014109 